\title{
Connecting Algols to CVs
}

\author{
M.M. Montgomery ${ }^{* \dagger}$ \\ University of Central Florida - Department of Physics, PSB 132, 4000 University Blvd., \\ Orlando, FL, USA \\ E-mail: montgomeryaphysics.ucf .edul
}

In this work, we review the evolutionary sequence of close binary systems, specifically the evolutionary sequence that connects Algols to Cataclysmic Variables. In support of this evolutionary sequence, we look for commonalities between Algols like $\beta$ Per and Cataclysmic Variables with accretion disks. One dynamical activity that seems to be present in both is prograde disk precession, which generates positive superhumps in light curves. To this list of commonalities, we suggest retrograde precession and negative superhumps, and we look for signatures in the Kepler light curves of WX Draconis, an Algol system like $\beta$ Per. From a comparison of light curves, we find that an accretion disk is likely present around the primary in WX Draconis. To generate the quasi-periodic long term modulation, this disk may be tilted and precessing in the retrograde direction.

The Golden Age of Cataclysmic Variables and Related Objects - III, Golden2015

7-12 September 2015

Palermo, Italy

* Speaker.

${ }^{\dagger}$ A footnote may follow. 


\section{Introduction}

Cataclysmic Variables (CVs) are close binary systems containing a white dwarf primary accreting gaseous material via an accretion disk from a low-mass main-sequence secondary star. Like $\mathrm{CVs}$, Algols are also interacting binaries. However, the stars in Alogls are much wider apart; and thus the system has a much longer orbital period. In addition, Algol-type binaries like $\beta$ Per (Algol) have a B-A-type main sequence star that is accreting gaseous material from a cool subgiant.

The evolutionary sequence tells us that the subgiant of this Algol type will evolve into a red giant that will lose its outer gaseous layers in a planetary nebula, leaving behind a remnant white dwarf that is in a close binary with a main-sequence secondary star. Thus, Algols like $\beta$ Per should evolve to become precursor CVs [e.g., Trimble (1983); Iben \& Tutukov (1985); Wheeler (2007)]. As such, studying both systems is necessary to find commonalities that support this evolutionary sequence. In this work, we discuss these commonalities, and we search for new ones.

In $\$ 2$, we list some commonalities between Algols like $\beta$ Per and CVs that have disks around their primaries. In $\S 3$, we provide some observational evidence in support of proposed, but unconfirmed, commonalities. In $\S 4$, we summarize and conclude.

\section{Current and Proposed Commonalities Between Algols \& CVs with Disks}

CVs are photometrically active, exhibiting variability on timescales of seconds to centuries [e.g., Warner (1995)]. For example, the CV dwarf novae (DN) SU UMa subclass experiences superoutbursts that can last for days, during which the system becomes significantly brighter. Of all in the CV DN SU UMa system, the accretion disk is the optically brightest object during the superoutburst. Therefore, the source to the increased luminosity from the system has to be due to an instability in the disk. One example is a tidal instability: As the disk grows in size from a ring, it expands both inwards and outwards until enough disk mass has expanded outward beyond the $m=3$ eccentric inner Lindblad resonance radius to be tidally forced by the secondary star into precessing in the prograde direction [see e.g., Whitehurst $(1988,1994)]$. Near superoutburst maximum, these tidal forces cause the disk to cyclically change shape from circular to eccentric and back to circular over a timeframe of about one orbital period. As a characteristic of the CV DN SU UMa system is periodic, large-amplitude, photometric modulations, which have a period that is a few percent longer than the orbital period [see e.g., Vogt (1982); Patterson $(1998,1999)$ ], these positive superhumps must be associated with this cyclic shape change of the disk.

In addition to positive superhumps and prograde precession, some disks around the primary in $\mathrm{CVs}$ generate negative superhumps and retrograde precession. Like positive superhumps, negative superhumps are also periodic and can have large-amplitude photometric modulations in the light curve. Negative superhumps, however, have a period that is slightly shorter than the orbital period. Like prograde precession, the disk's rotation axis wobbles. However, in retrograde precession, the wobble direction is opposite that of orbital motion. To achieve retrograde precession and to generate signals of negative superhumps in periodograms or Fourier transforms, numerical simulations (Montgomery 2012) show that the disk must leave the orbital plane (i.e., tilt) by at least a few degrees (Montgomery 2009). A likely source to disk tilt around the line of nodes is lift (Montgomery $\&$ Martin 2001). 
Like CVs, Algols are also dynamically active. For example, Retter et al. (2005) found periodicities in radio light curves from two Algol systems that suggest that these $\beta$ Per type systems have disks that precess in the prograde direction. Their prediction is that high-resolution light curves should indicate positive superhumps. In 2010, Peterson et al. (2010) confirmed the presence of positive superhumps in these Algol types. Additional confirmation of well-defined accretion disks is from 2D Doppler tomography [see e.g, Richards et al. (1995); Richards (2004); Richards et al. (2014)]. From 3D Dopper tomography, distributions of gas flows are found out of the orbital plane, indicating that the accretion disk may be tilted and precessing in the retrograde direction [see Richards et al. (2012)]. If the disk is tilted and precessing, we propose that the light curve and/or periodogram should indicate the presence of negative superhumps.

As both non-magnetic CVs and Algols like $\beta$ Per have confirmed accretion disks, confirmed positive superhumps, and confirmed prograde precession, positive superhumps and prograde precession are on the list of current commonalities between these systems. Although negative superhumps and retrograde precession have not yet been confirmed in these Algol types, negative superhumps and retrograde precession are likely contenders for the list. Confirmation though may need higher resolution light curves, such as those from the Kepler/K2 space telescope.

\section{Evidence in Support of Retrograde Precession and Negative Superhumps}

As discussed in the previous section, negative superhumps and retrograde precession are suggested commonalities connecting Algols of the $\beta$ Per type with CVs that are known to precess in the retrograde direction and have negative superhump in their light curves. Possibly the reason why negative superhumps and retrograde precession have not yet been confirmed is due to the resolution of the light curve. Historically, ground-based telescopes have not been able to detect the weak modulation in CV light curves that indicate the disk is precessing in the retrograde direction. Thus, finding this modulation in Alogls using ground-based observations becomes even more difficult. As such, we turn to Kepler/K2 light curves as space-based observations can continuously monitor and produce photometry with high accuracy and increased resolution.

The Algol target of choice is WX Draconis, which is a short-period, eclipsing Algol binary of the $\beta$ Per type. According to Budding (2004), its orbital period is 1.80 days and it has an A8 main sequence star in a binary with a $\mathrm{K} 0$ subgiant.

Figure 1 shows a composite light curve from Kepler Quarters Q10-Q12 and Q14-Q16 of WX Draconis. Each consecutive three quarters has been arbitrarily selected from the list of all observed Kepler quarters. As indicated by the side-by-side red and black arrows, the total flux during consecutive out-of-secondary and out-of-primary eclipse phases varies from quarter to quarter. Sometimes the total out-of-eclipse flux is the same (parallel side-by-side red and black arrows) and sometimes one or the other is more or less. Nonetheless, a long-period (hundreds of days) modulation is present in each set of three consecutive Kepler quarter light curves, as well as in the other quarters not shown here.

The long-term variability shown in Figure 1 can be fit with two models by Peters \& Wilson (2014) - either an accretion hot spot on the primary that jumps in longitude or a rather large magnetic cool spot region on the secondary. In this work, we add to the list a third gravitationally bound body and a fourth possibility of a precessing disk around the primary. Although a hot spot is 

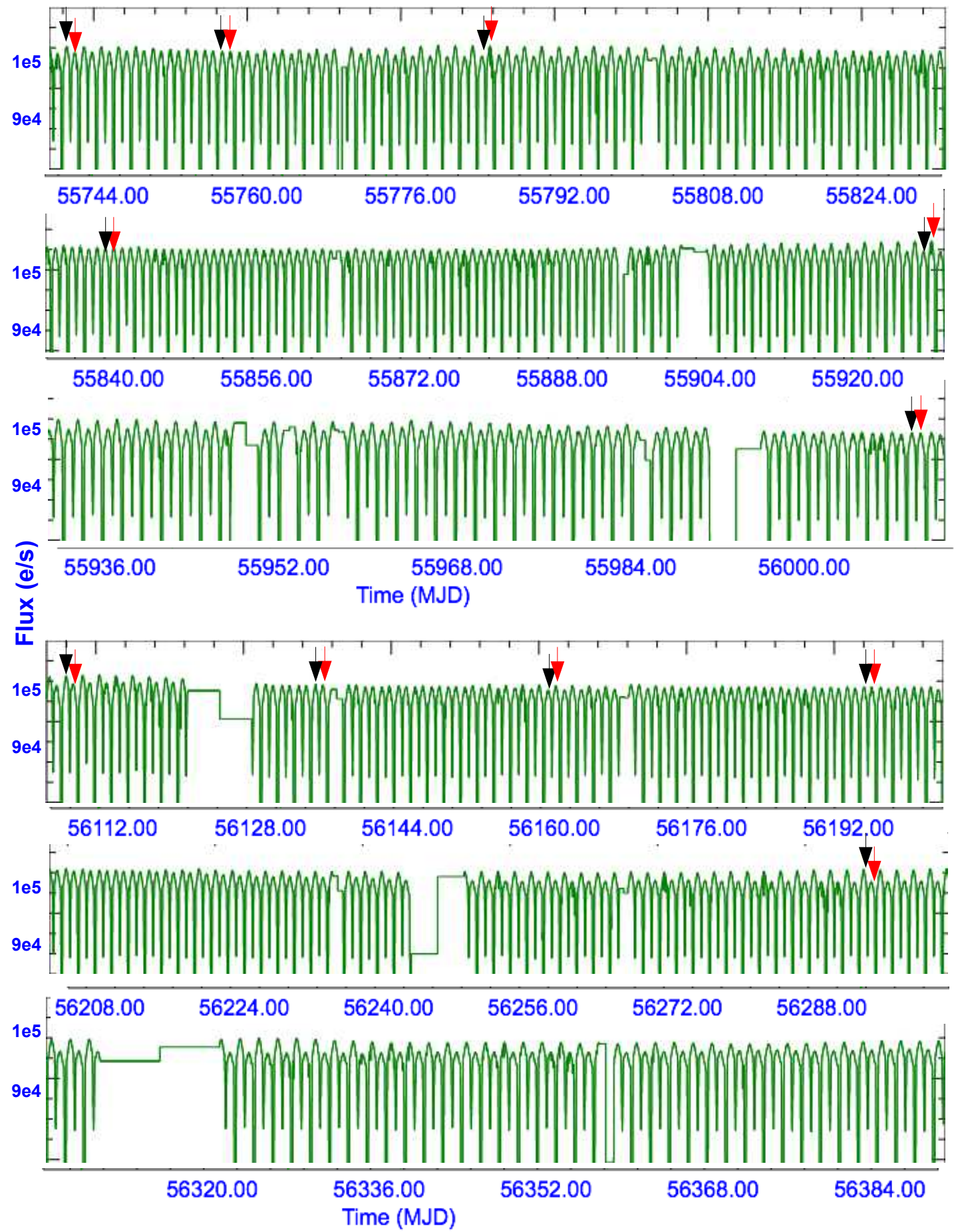

Figure 1: Composite light curve of WX Draconis showing $30^{m}$ long cadences for Kepler Q10, Q11, Q12 and Q14, Q15, Q16, respectively. from top-to-bottom. All have been retrieved from MAST. Side-by-side black and red arrows point to the varying total flux during consecutive out-of-secondary and out-of-primary eclipse phases. 
a likely source (but not necessarily directly striking the photosphere of the primary), a reasonable explanation for the longitudinal jumping on the photosphere of the primary cannot be found and is not provided by Peters \& Wilson (2014). A single, large region of cool spots on the secondary does not seem highly probable for many reasons, including that star spot lifetimes are short yet the modulations are present in all Kepler light curves and are of long-period. A third body can also be eliminated as its orbital period and mass (Zasche et al. 2015) would have to be 14 years and $0.0027(9) M_{\odot}$, respectively, or not massive or close enough to torque the system to generate the long-period modulation [see e.g., Montgomery (2009b)]. In addition, Kepler MAST has identified WX Draconis as a false positive. Of the possible sources, we suggest that a tilted precessing disk is most likely.

In support of a tilted, precessing disk as a potential source, we turn to the short cadence light curve generated in Peters et al. (2013). As shown, the flux from the system during out-of-eclipse is not constant, and the shape of the light curve is very similar to that from $\beta$ Per in the red to infrared wavelengths as shown in Figure 3.

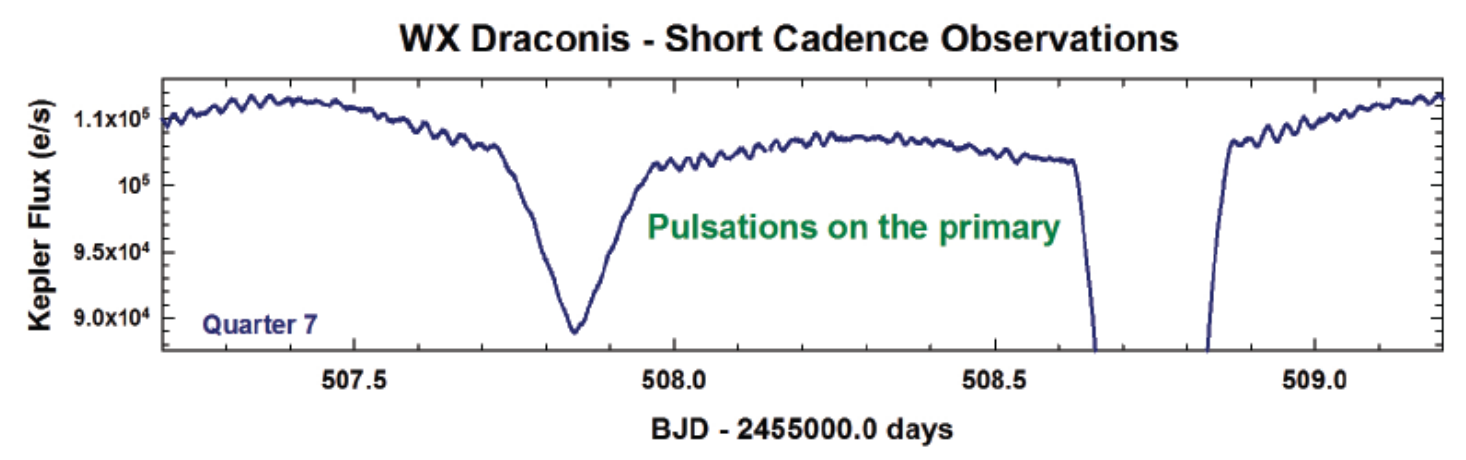

Figure 2: Short cadence Kepler Q7 light curve of WX Draconis from Peters et al. (2013).

As $\beta$ Per is known to have a disk around its primary and as the out-of-eclipse curvatures in the light curve suggest an additional light source other than the primary and the secondary, a logical choice to the additional light from WX Draconis is an accretion disk. In addition, as the out-ofeclipse curvature is more apparent in the infrared wavelengths, the disk around the primary in $\beta$ Per (and thus WX Draconis) may likely contain dust. To these hypotheses, we add that the disk in WX Draconis is likely tilted and precessing in the retrograde direction. A tilted disk that is precessing in the retrograde direction could logically explain the long period modulation in Figure 1. As the retrograde precession period depends upon the degree of disk tilt [see Montgomery (2009)], a nonconstant degree of disk tilt could explain the quasi-periodic nature of this long period modulation: A hot spot striking the face of a disk that varies its disk tilt, and thus varies in its period of retrograde precession, could explain the longitudinal jump found by Peters et al. (2013). Further support is a comparison of analytical modeling to observations: Using the system parameters found by Retter et al. (2005) and the analytical expressions in Montgomery (2009), we find that a tilted disk in the $\beta$ Per system would precess in the retrograde direction approximately once every $\sim 95$ days, a period that is of the right order of magnitude seen in Figure 1. 


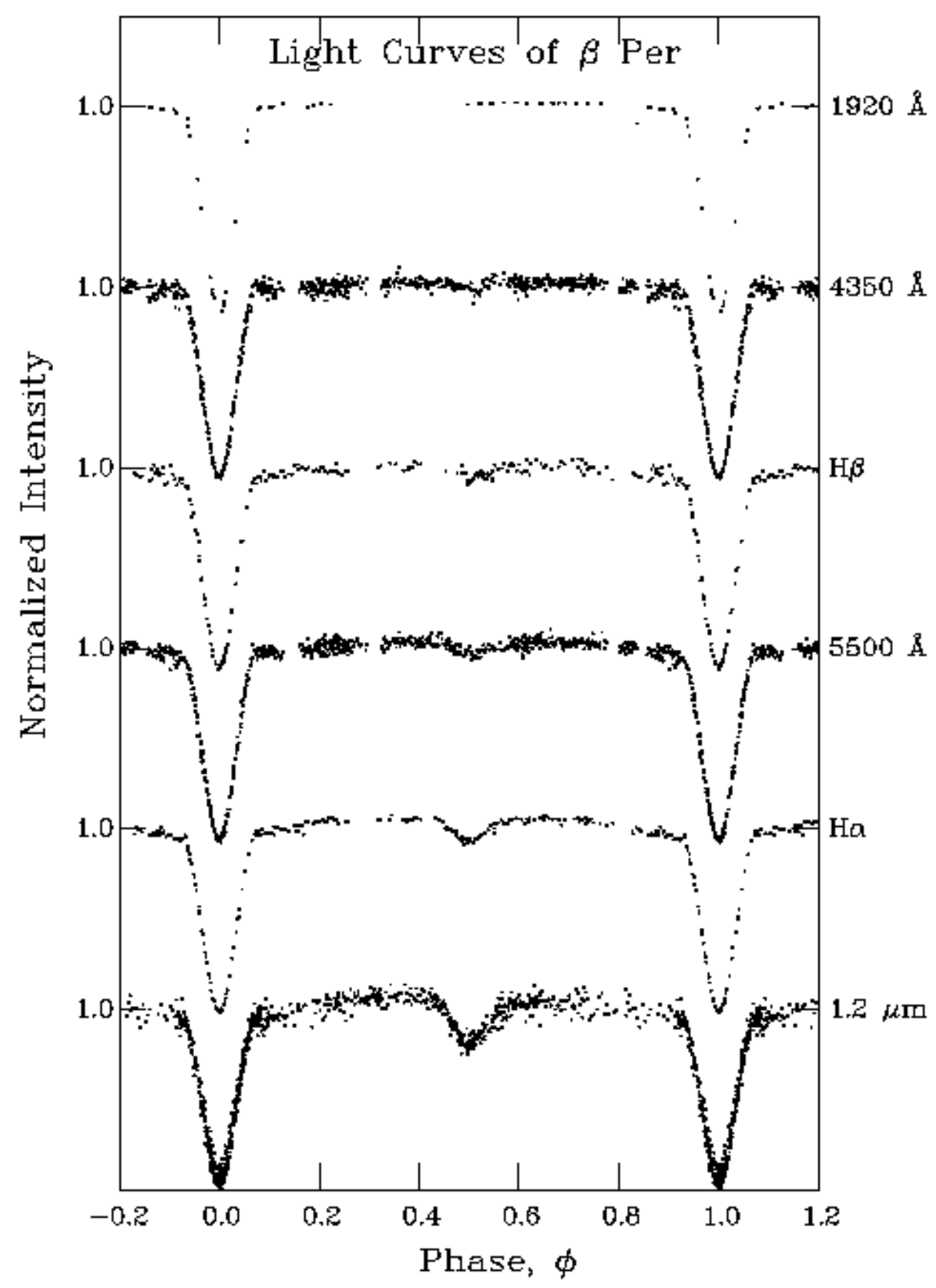

Figure 3: Light curves of $\beta$ Per from UV-to-IR wavelengths (top-to-bottom), courtesy of Mercedes Richards (private comm.). 


\section{Conclusions}

In this work, we review the evolutionary sequence connecting Algols to CVs. Observations that support this evolutionary sequence are disks that accrete and precess in the prograde direction, which generates positive superhumps in their light curves. To this, we argue that retrograde precession, which generates negative superhumps in the light curves, should also be contenders. From a comparison of light curves from Algols known to have accretion disks (i.e., $\beta$ Per) and shortcadence Kepler light curves from Algols of a similar type (i.e., WX Draconis), we argue that WX Draconis also has an accretion disk around its primary. Further, we propose that the quasi-periodic, long-period modulation seen in long cadence Kepler light curves is due to a disk that tilts of varying degree, and thus precesses of varying period in the retrograde direction, which could explain the longitudinal jump found by Peters \& Wilson (2014).

\section{References}

[Budding(2004)] Budding, E. et al. 2004, A\&A, 417, 263

[Iben \& Tutukov(1985)] Iben, L. \& Tutukov, A. V., 1985, ApJS, 58, 661

[Montgomery(2009)] Montgomery, M. M. et al. 2009, mnras, 394, 1897

[Montgomery(2009b)] Montgomery, M. M. et al. 2009b, apj, 705, 603

[Montgomery(2012)] Montgomery, M. M. et al. 2012, apj, 745, L25

[Montgomery \& Martin(2010)] Montgomery, M. M. \& Martin E. L. 2010, apj, 722, 989

[Patterson(1998)] Patterson, J. 1998, pasp, 110, 1132

[Patterson(1999)] Patterson, J. 1999, in Disk Instabilities in Close Binary Systems, ed. S. Mineshige \& J. C. Wheeler (Tokyo: Universal Academy Press), p. 61

[Peters et al.(2013)] Peters, G. et al. 2013, Second Kepler Science Conference, NASA Ames, Poster 2-202 [Peters \& Wilson(2014)] Peters, G. \& Wilson, R. E. 2014, AAS, 22314602P

[Peterson et al.(2010)] Peterson, W. M. et al. 2010, Nature, 463, 207

[Retter et al.(2005)] Retter, A. et al. 2005, apj, 621, 417

[Richards et al.(1995)] Richards, M. T. et al. 1995, apj, 438, L103

[Richards(2004)] Richards, M. T. 2004, AN, 325, 229

[Richards et al.(2012)] Richards, M. T. et al. 2012, apj, 760, 8

[Richards et al.(2014)] Richards, M. T. et al. 2014, apj, 795, 160

[Trimble(1983)] Trimble, V. 1983, Nature, 303, 137

[Vogt(1982)] Vogt, N. 1982, apj, 252, 653

[Warner(1995)] Warner, B., 1995, Cataclysmic Variable Stars (Cambridge: Cambridge University Press)

[Wheeler(2007)] Wheeler, J.-C., 2007, Cosmi Catastrophes (Cambridge: Cambridge University Press)

[Whitehurst(1988)] Whitehurst, R. 1988, mnras, 232, 35

[Whitehurst(1994)] Whitehurst, R. 1994, mnras, 266, 35 
[Zasche et al.(2015)] Zasche, P. et al. 2015, aj, 149, 197

\section{DISCUSSION}

Hannes Breytenback: In the Kepler data of WX Dra, the relative depth of the secondary eclipse varies along with the relative peak brightness. Is this consistent with your interpretation of the system containing a disk?

MICHELE M. MONTGOMERY: Yes. A disk that varies in degree of disk tilt will have a varying precession period and will have a varying degree of brightness relative to the observer.

Dmitry Bisikalo: What is the size of the accretion disk in your model of WX Dra? What is the source of precession?

MICHELE M. MONTGOMERY: The light curve indicates an optically thin, but large disk. More observations are needed at different wavelengths (e.g., infrared) to better determine. The source to precession is tidal torques on a disk that is tilted around the line of nodes. 